\title{
Characterization of 400 Volt High Impedance Fault with Current and Magnetic Field Measurements
}

\author{
Anwarul Islam Sifat, Graduate Member, IEEE, Fiona J Stevens McFadden, Member, IEEE, Joseph Bailey, \\ Ramesh Rayudu, Senior Member, IEEE, and Arvid Hunze
}

\begin{abstract}
Electrical faults, which can occur at all voltage levels in an electricity supply system, are a health and safety risk. Multi-branch distribution networks represent a significant ongoing challenge for fault detection, with the greatest challenge being high impedance fault (HIF) detection. To date, research has focused on higher voltage levels, and fault monitoring sensors have traditionally only been installed in limited locations within the higher voltage networks. The main contributions of this paper are to characterize a high impedance fault (HIF) involving a tree branch and to experimentally verify the feasibility of giant magneto-resistive (GMR) sensors, located distant from the overhead lines, for fault detection. In a purpose-built $400 \mathrm{~V}$ physical simulation test facility, we have collected current and magnetic field data during HIF involving a tree branch. We have identified new characteristics in the early stages of this fault type, which persist for a reasonable length of time but are only observable when suitable signal processing techniques are applied. New detection schemes will, therefore, need to be developed to detect such faults. GMR sensors were found to be suitable for observing the characteristics of HIF, validating their potential use for fault detection.
\end{abstract}

Keywords-High Impedance Fault, Indoor Test Facility, Test Facility Design, Distribution Network, 400 V, Fault Data.

\section{INTRODUCTION}

Detection of power system faults is of great importance to electricity distribution system operators and detecting HIFs are an ongoing industry and research challenge. Furthermore, the increasing complexity of networks are introducing numerous new challenges and threats that would benefit from more widespread continuous monitoring, control and optimization [1].

Electrical faults are generalised into two types: Low and High Impedance faults. A low impedance of the fault medium generates a high current and gives the low impedance fault (LIF) its name. The conventional over-current relay and/or fuse detects the LIF incident and isolates the circuit from being damaged. In contrast, according to the IEEE power system relay committee, HIF, "do not produce enough fault current

A. I. Sifat and R. Rayudu are with Department of Engineering and Computer Science Victoria University of Wellington. email:anwarul.sifat@vuw.ac.nz, ramesh.rayudu@vuw.ac.nz

F. J. Stevens McFadden and J. Bailey are with Robinson Resaerch Institute, Victoria University of Wellington. email: fiona.stevensmcfadden@vuw.ac.nz, joseph.bailey@vuw.ac.nz

A. Hunze is with Research Flow NZ Limited, Lower Hutt, New Zealand. email: arvid@grantconsultingnz.com

This work was financially supported in part by the New Zealand Science for Technological Innovation National Science Challenge (contract RTVU1702); Ministry of Business, Innovation and Employment New Zealand (contract RTVU1811) and Victoria University of Wellington Research Trust.

Corresponding author: A. I. Sifat to be detectable by conventional overcurrent relays or fuses" [2]. Typically, in these HIF faults an energized conductor is in contact with a quasi-insulated object (e.g. a tree branch, soil). The sensitivity of a conventional relay is inadequate to detect such low fault currents. However, undetected and energized downed conductors pose a safety risk to people and the arcing phenomena associated with an HIF are hazardous. HIF have been implicated in several incidents leading to bush fire, unexpected accidents and human death [3], [4].

The characterization of fault behaviour is a prerequisite for building an optimal detection scheme. The oscillatory and impulsive behaviour of fault transients varies with the self and mutual capacitance, impedance and types of connected load [5]. For overhead lines, these parameters are directly related to the voltage level [6]. Therefore, the characteristics of faults and their impact on the alternating current (AC) are a function of the level of voltage, current and system frequency.

In $11 \mathrm{kV}$ distribution networks, low-impedance high-current faults (LIF) are detectable using a substation-based fault tripping mechanism or fault passage indicators (FPI) placed within the network. However, at the consumer end, the $400 \mathrm{~V}$ distribution lines are electrically separated from the medium voltage part of the system due to the distribution transformer configuration, so faults in the $400 \mathrm{~V}$ networks can not readily be observed by the devices in the higher voltage part of the network. LIFs at this level are mainly protected only by a fuse, however, these are not triggered by low-current highimpedance faults (HIF). Fault incidents in the $400 \mathrm{~V}$ level, therefore, largely remain obscured [7], [8] due to lack of monitoring sensors at this level. Currently, HIF research has focused on the medium voltage $(>1 \mathrm{kV})$ networks [9], [10], [11]. Research to characterize faults at low-voltage $(400 \mathrm{~V})$ level is therefore important and will allow development of a practical and efficient fault detection technique.

Sensors are a key element for monitoring the electricity network status and the basis for fault detection. To date, fault detection schemes being investigated have primarily utilized current and/ or voltage measurements. This requires multiple sensors to be installed at each measuring location as it is a poly-phase circuit. This is economically prohibitive for widespread multi-branch distribution networks. Traditionally, the current transformer (CT) has had a predominant role in current sensing and fault detection. However, they are not ideal as a more widespread smart grid sensor due to their intrinsic characteristics, e.g., their non-linearity, narrow bandwidth, and lack of capability for direct current (DC) measurement [12], [13]. 
Magneto-resistive (MR) sensors are attractive due to their non-contact sensing ability, measurement accuracy, miniaturesize, and low-cost [14], [15], which would allow easy installation on overhead network poles without additional costs. Therefore, the wide-area monitoring of a typical multi-branch distribution network could be feasible using MR sensors.

More recently, magnetic sensors as a non-invasive fault current sensing device have become popular to researchers. An actual sensor based fault detection scheme was experimentally evaluated in [16]. An array of tunnel magneto-resistive sensors (TMR) was placed in the immediate vicinity $(10 \mathrm{~mm})$ of overhead transmission lines in a reduced-scale laboratory experiment. The proposed scheme was designed to detect the magnetic field distortion due to the fault current traveling wave. The study did not evaluate if the sensors could be placed at a distance from the overhead lines, nor was the response under non-linear types of faults, for example HIF evaluated. In [17], experimentally collected $20 \mathrm{kV}$ HIF current data was converted to magnetic signatures using a mathematical model. The model approximates the magnetic field measured at a distance from overhead lines. However, this research did not address the response of measurements from an actual magnetic sensor under the fault conditions.

In summary, research on the subject of magnetic sensors for fault detection has been mostly limited to reduced scale laboratory experiments and model-based magnetic field calculation. Therefore, proper experimental HIF evaluation are required to verify MR sensor's effectiveness as opposed to traditional current sensors.

The study presented in this paper therefore had two primary objectives: 1. Investigate the characteristic of HIF faults in a low voltage $(400 \mathrm{~V})$ distribution system, using a purpose-built physical network and fault simulation test facility; 2 . Evaluate the fault phenomena observable in measurements using GMR sensors as compared to current measurements, in both raw signals and Fast Fourier Transforms and Hilbert Transforms.

In this study, a tree branch HIF was fully characterized in a $400 \mathrm{~V}$ network using non-contact GMR sensors placed at a distance from overhead lines, along with phase and fault return path voltage and current measurements. This paper helps fill the gap in knowledge of high impedance faults in both the current and magnetic domains. The results also verify the feasibility of using non-contact GMR sensors to monitor and detect $400 \mathrm{~V}$ overhead lines faults compared to Hall effect sensors.

\section{Methodology}

Actual fault current data contains complex behaviour influenced by natural elements which cannot be realized via computer aided simulations [18], therefore, in this study we have experimentally characterized HIF in $400 \mathrm{~V}$ networks versus other common network activities. The experimental work was conducted a $400 \mathrm{~V}$ physical simulation test facility that we have designed and implemented.

A schematic of the test facility is shown in Figure 1. This test facility has been comprehensively detailed in a separate paper [19], but a summary of the important details for each component of the experimental setup is given below. A description of the experiments performed is also given in section II-C.

\section{A. Power Section}

The source of power in the facility is an isolating transformer. The internal winding of the transformer (vector group: Dyn11) is configured to isolate the primary winding from the secondary winding. A bolted fault at the secondary is therefore isolated from the existing primary electrical subsystem. The arrangement is there to protect the source (primary) side from unwanted tripping during the experiments.

\section{B. Overhead Line Section}

Actual overhead distribution lines were replicated inside the test facility. The length of the distribution lines was scaled down to accommodate them inside the facility. This section was included as the response of the GMR sensor relies on the spatial features of overhead lines. A mathematical model was utilized to calculate the magnetic field generated from overhead lines and numerical analysis verified that $80 \%$ of the magnetic field generated by the typical $30 \mathrm{~m}$ pole to pole span of overhead line networks is generated by the $3 \mathrm{~m}$ span length used in the test facility. The detailed analysis for this was discussed in [19].

In addition to this spatially equivalent overhead line segment, the remaining typical $600 \mathrm{~m}$ circuit line length was physically modelled in $100 \mathrm{~m}$ increments using a transmission line $P i$ model [20]. This physical model is a combination of lumped components for which values were collected via a survey on NZ distribution networks [21].

The third section of the test facility incorporates a central fault, load remote control and data acquisition system (DAQ).

\section{Load Section}

The load section of the experimental setup included two types of loads (Fig. 2): heaters and an outdoor light unit. These are commonly used household loads. For the heaters we have used both radiant and fan heaters. Therefore, overall the loads are a mix of resistive and inductive elements.

\section{Sensor and Data Acquisition Unit}

The data acquisition unit collects the data from voltage, current and magnetic sensors during the fault experiment. We used Hall-effect current sensors (LEM HO 25-NP) [22] to continuously monitor the 3-phase currents along with the neutral, rather than CTs as they provide more accurate current measurements. A voltage divider circuit assembled on a printed circuit board (PCB) was used to acquire voltage data from the setup. We have used two data acquisition modules or DAQ units from National Instruments [23]. The two DAQ modules are time synchronized using a master slave configuration [24]. The data was collected through the DAQ synchronously at $28 \mathrm{kHz}$ sampling rate.

For the magnetic sensors, we have designed and developed a 3D GMR sensor head (Fig. 3 (a)). Three single axis NVE 


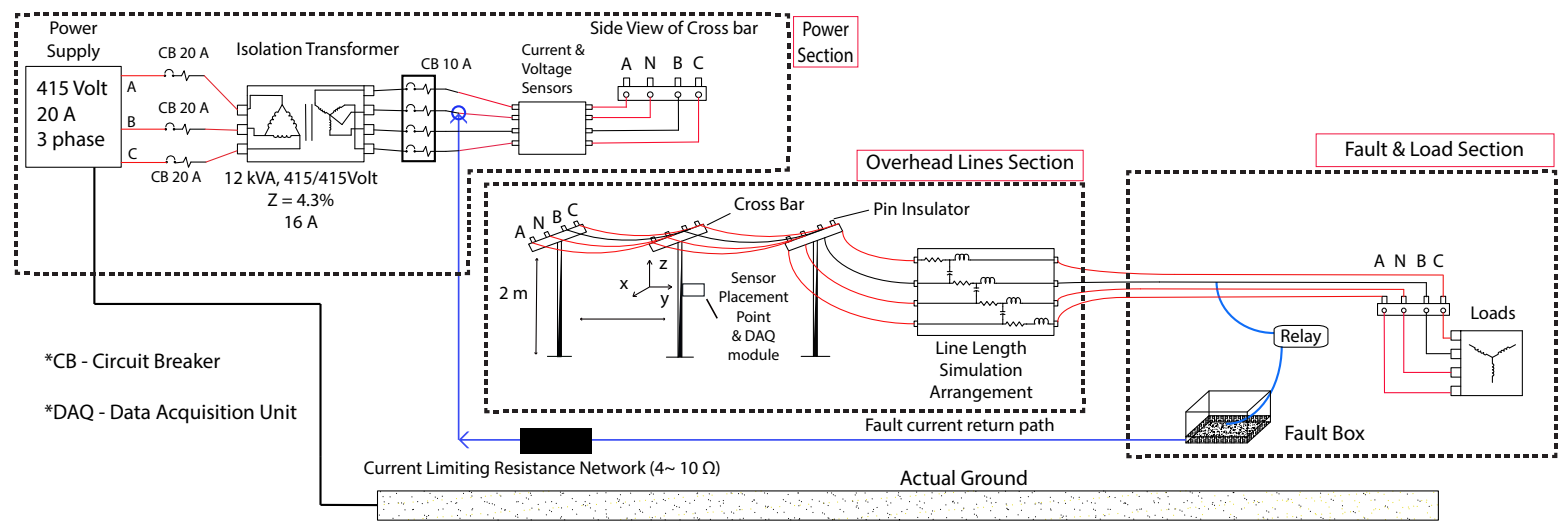

Fig. 1. Test Facility Schematic. The blue line indicates the fault current return path connected to transformer neutral.

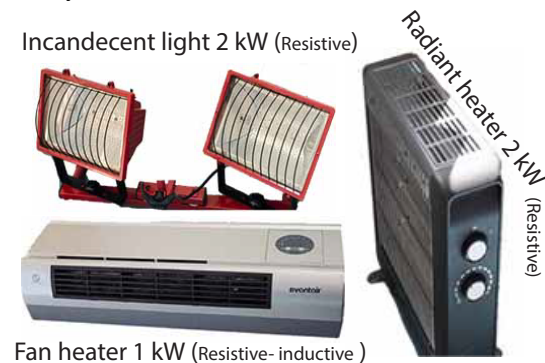

Fig. 2. Types of loads used in the experiments. Incandescent light, radiant heater and fan heater are connected with phase A, B and C respectively.

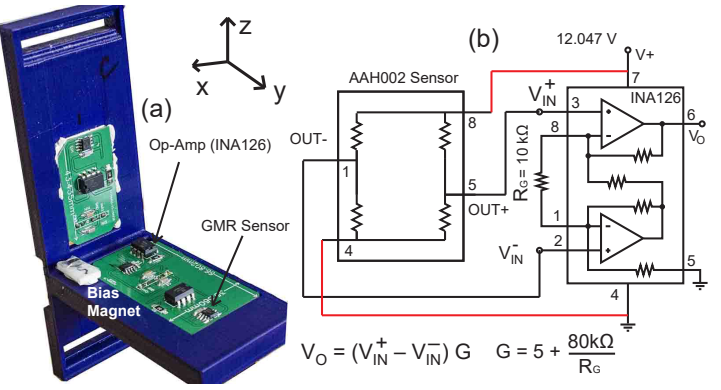

Fig. 3. (a) GMR sensor head; (b) Circuit assembly schematic of a GMR sensor

AAH002 sensors with am average sensitivity of $14.5 \mathrm{mV} / \mathrm{V}$ Oe [25] are powered by a 12-volt DC source and are placed on two PCB boards (Fig. 3 (b)). The sensitive axis of each sensor is directed towards one of the $x, y$ and $z$ directions to make a 3 -axis sensor. The sensor head was placed $0.6 \mathrm{~m}$ below the overhead lines in the center of the four lines.

The output signal from each of the 3-axis sensors is the combination of all four conductors. Mathematically [26], [27],

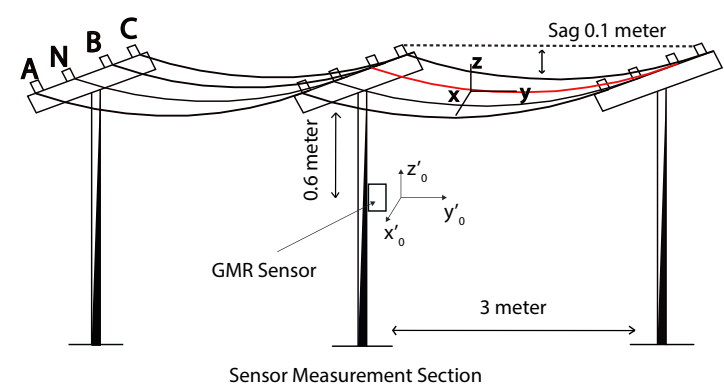

Fig. 4. Test facility overhead line geometry.

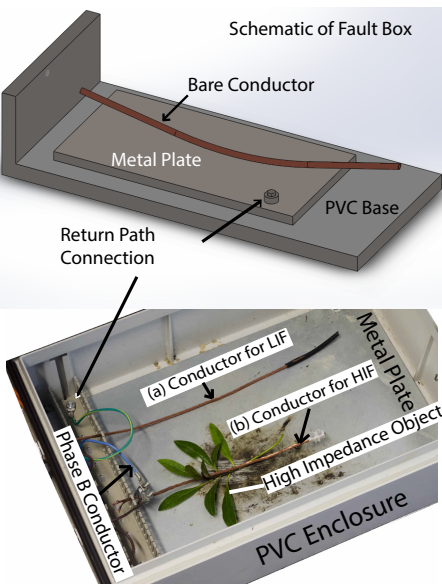

Fig. 5. Fault staging apparatus (Fault Box) showing setup of (a) LIF; (b) HIF with a tree branch used as a high impedance object (bottom figure).

this combined signal can be expressed as,

$$
\begin{aligned}
& \overrightarrow{B_{x}}=\frac{\mu_{0} I}{4 \pi} \int_{-D / 2}^{D / 2}\left[\frac{z_{0}^{\prime}-\frac{1}{c}(\cosh (c y)-1)+\left(y-y_{0}^{\prime}\right) \sinh c y}{\left|\left(x_{0}^{\prime}-x\right) \hat{i}+\left(y_{0}^{\prime}-y\right) \hat{j}+\left(z_{0}^{\prime}-\frac{1}{c}(\cosh (c y)-1)\right) \hat{k}\right|^{3}} \hat{i}\right] d y \\
& \overrightarrow{B_{y}}=\frac{\mu_{0} I}{4 \pi} \int_{-D / 2}^{D / 2}\left[\frac{\left(x_{0}^{\prime}-x\right) \sinh c y}{\left|\left(x_{0}^{\prime}-x\right) \hat{i}+\left(y_{0}^{\prime}-y\right) \hat{j}+\left(z_{0}^{\prime}-\frac{1}{c}(\cosh (c y)-1)\right) \hat{k}\right|^{3}} \hat{j}\right] d y \\
& \overrightarrow{B_{z}}=\frac{\mu_{0} I}{4 \pi} \int_{-D / 2}^{D / 2}\left[\frac{-\left(x_{0}^{\prime}-x\right)}{\left|\left(x_{0}^{\prime}-x\right) \hat{i}+\left(y_{0}^{\prime}-y\right) \hat{j}+\left(z_{0}^{\prime}-\frac{1}{c}(\cosh (c y)-1)\right) \hat{k}\right|^{3}} \hat{k}\right] d y
\end{aligned}
$$

The current through the power line is flowing in the $y$ axis direction (Fig. 4), therefore, $B_{y} \approx 0$. The $x$-axis is in the direction of the cross-arm. The conductor sag is in the $z$ direction and is defined by,

$$
z=1 / c \times(\cosh (c y)-1) ;-D / 2 \leq y \leq D / 2
$$

where, $c$ is the curvature of the overhead line conductor. The ratio of per unit length conductor weight $w(\mathrm{~kg})$ and horizontal tension $H$ together define $c$, i.e. $c=w / H$. The horizontal tension is given by, $H=w D^{2} / 8 S$; where, $D=$ length of the conductor (m), $S=$ sag length (m).

Typically, both the GMR and Hall-effect sensors give a voltage output for the current and magnetic field changes. The voltage output of the Hall-effect sensor is multiplied by the sensitivity factor $H_{s n s}(\mathrm{mV} / \mathrm{A})$ to calculate the current in the 
conductors. In our setup, the GMR sensor output is amplified by an instrumentation amplifier. Thus, the output is divided by the amplifier gain to get the actual voltage output. The output voltage is then converted to magnetic flux density using (5),

$$
B(\phi)=V_{s n s} \times\left[\frac{1}{\left(\frac{V_{o p} \times S_{n s}}{100}\right) \times 10^{3}}\right]
$$

Where, Sensor Output Voltage $V_{s n s}(\mathrm{~V})$; Operating Voltage $V_{o p}(\mathrm{~V})$; Sensitivity of the sensor $S_{n s}(\mathrm{mV} / \mathrm{V}-\mathrm{Oe})$; Magnetic Flux Density $B(\phi)(\mathrm{T})$.

\section{E. Fault Simulation Procedure}

A fault staging apparatus was constructed to simulate faults in the facility (Fig. 5). The apparatus is a plastic box with a metal plate attached as a base. The metal plate is connected to the transformer neutral via a current limiting resistor network. The arrangement creates a short circuit path for current to travel from the phase $B$ conductor to the neutral point of the transformer. Usually, a short circuit could produce more than 1 $\mathrm{kA}$. However, a network of resistors connected in series with the return path was included to limit the fault current to a maximum of $27 \mathrm{~A}$ (RMS) for protection of the test facility components.

The fault box also contains two alternative (manually switchable) bare conductors connected in parallel with phase $B$. One for LIF experiments and the other with a quasiinsulating object between the conductor and metal plate for HIF experiments. A relay switch is connected between the bare conductor in the box and phase $B$. The relay is controlled by the central control software designed as graphic user interface (GUI) via LabView code.

We have performed high impedance faults on a tree branch, sea-sand, and clay soil. The widest range of HIF phenomena observed was in the fault experiments involving the tree branch. For brevity, therefore, this paper reports on the results of just these tree branch experiments. HIF faults on other surface materials will be reported in a future paper.

For the tree branch HIF experiment the bare conductor was placed on a green tree branch (Fig. 5 (b)) in the fault simulation box. A common species of tree in New Zealand called Whitey Wood or Acradenia frankliniae was used. Since, the tree branch has a non-linear high impedance, it produces a low fault current.

The experimental procedure began with energizing the isolation transformer. Then via LabView GUI, the loads (section II-C) are remotely turned on, one at a time. Once the system loading was stabilized (after approximately 3s), the fault was then initiated automatically via a switching relay at 10 s after the data acquisition starts saving the data from sensors. The HIF experiment was terminated via the GUI after typically 4050 s.

\section{Signal Characterization in Frequency AND TIME DOMAIN}

To fully explore the characteristics of the tree-branch HIF in the time and frequency domains we have analyzed the measured data using Fast Fourier and Hilbert Transforms. The

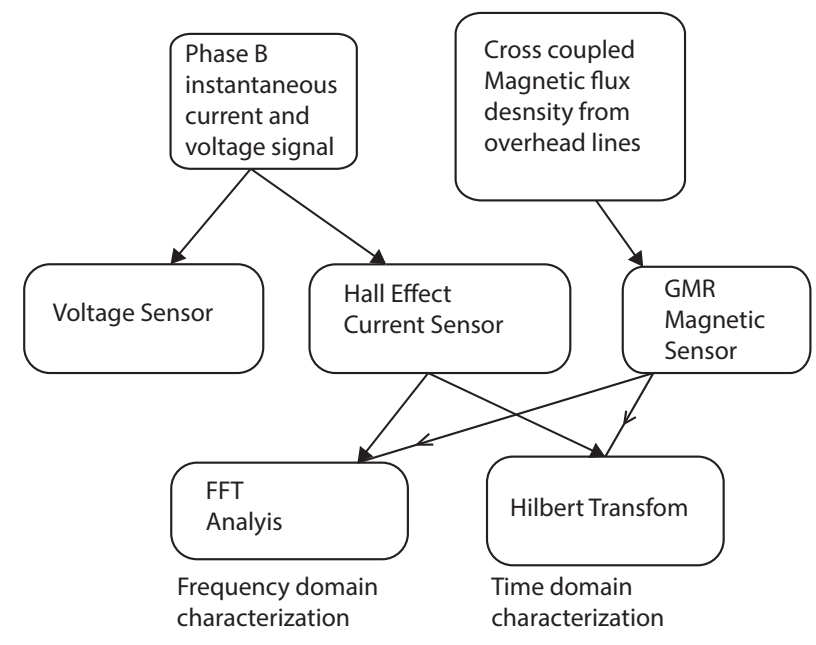

Fig. 6. Steps in characterization of current and magnetic field signals in the time and frequency domains.

$50 \mathrm{~Hz}$ component was filtered out of the current and magnetic field signals to focus on the higher frequency transient impulses embedded in the signals.

\section{A. Fast Fourier Transform (FFT) Analysis}

Real-world waveforms can be a combination of multiple sine-waves of different frequencies, amplitude, and phase angle. Fourier analysis is one of the standard procedures for determining the frequency content of real-world signals and their stationary and transient phenomenon [28].

\section{B. Hilbert Transform (HT) Analysis}

Any real sinusoidal signal e.g. $A \cos (\omega t+\phi)$ can be represented by a (positive frequency) complex sinusoid i.e. $A e^{j(\omega t+\phi)}$ by generating a phase quadrature component. Thus,

$$
A e^{j(\omega t+\phi)}=A[\cos (\omega t+\phi)+j \sin (\omega t+\phi)]
$$

where, $A \sin (\omega t+\phi)$ is the "imaginary part". The in-phase component can be transformed into the phase quadrature by a quarter cycle time shift [29]. This is called the Hilbert transform filter. The filter shifts each sinusoid component by a quarter cycle.

The Hilbert transfer function in the time domain is,

$$
h(t)=\frac{1}{\pi t} ;-\infty<t<\infty
$$

The signal $\frac{1}{\pi t}$ has a Fourier transform. Therefore, in the frequency domain the Hilbert transform is,

$$
H(f)=-j \operatorname{sgn}(f)= \begin{cases}-j & f>0 \\ 0 & f=0 \\ j & f<0\end{cases}
$$

Here, sgn denotes the expression of sign function.

For an arbitrary signal $g(t)$ the Hilbert transform is defined as,

$$
H\left\{g^{\prime}(t)\right\}=g(t) \times h(t)=g(t) \times \frac{1}{\pi t}
$$

The time derivative of this equation yields,

$$
H\left\{g^{\prime}(t)\right\}=\frac{1}{\pi} \int_{-\infty}^{\infty} \frac{g(\tau)}{t-\tau} d \tau
$$




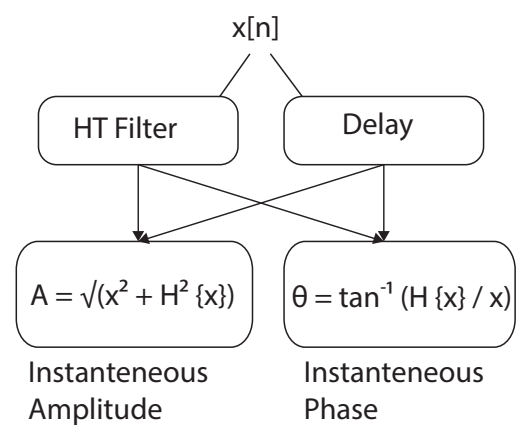

Fig. 7. Steps in HT to compute the instantaneous amplitude and phase angle.

The integral in 10 is an improper integral. Thus, the Hilbert transform is defined using the Cauchy principal value of the integral in 10 , whenever, the value exists.

$$
H\left\{g^{\prime}(t)\right\}=\frac{1}{\pi} \zeta \int_{-\infty}^{\infty} \frac{g(\tau)}{t-\tau} d \tau
$$

Where, $\zeta=$ Cauchy principal value [30].

If the signal $g(t)$ has a Fourier transform $G(f)$ then,

$$
H(f)=-j \operatorname{sgn}(f) G(f)
$$

Here, $-j \operatorname{sgn}(f)$, shifts the negative frequency components $G(f)$ by $\frac{\pi}{2}$ and positive frequency components by $-\frac{\pi}{2}$. The computation can be mathematically explained by considering an analytical signal $k(t)$ composed of a real signal $x(t)$ and HT shifted by $\frac{\pi}{2}$, defined as $k(t)=x(t)+j H\{x(t)\}$. This can be expressed in exponential notation, $x_{A}(t)=A(t) e^{j \phi(t)}$, where, $A(t)$ instantaneous amplitude and $\phi(t)$ instantaneous phase.

Typically, in a real world application the negative frequency components are redundant. So, the HT can be considered as an all pass filter [31].

In this study, the current and magnetic field signals were individually passed (Fig. 6) to the HT filter block composed of a finite impulse response linear phase filter of order 30th, which was designed using the Parks-McClellan algorithm [32] and implemented in Matlab by means of frequency component shifting to satisfy 12 . The input is also delayed to compensate the delay generated by the HT block. The parallel output from the HT filter and delay block are squared and summed to get the instantaneous amplitude. The instantaneous phase or phase angle is arithmetically computed by inverse tangent (Fig. 7).

The objective in performing a HT was to extract and highlight the features of fault transients. When applied to a pure sinusoidal waveform, the instantaneous phase would change linearly in a saw-tooth shape over a single cycle [33]. However, when a signal is a combination of numerous sinusoidal waveforms or high frequency noise, the phase angle does not change linearly over a single cycle [33]. Therefore, noise can be observed in the instantaneous phase. The phase angle noise is inversely proportional to the magnitude of the signal to noise ratio [34].

\section{Experimental Results from High Impedance FAULT EXPERIMENT}

The characteristics of any fault mostly depends on the fault surface material. An HIF occurs when a conductor is in contact with a high impedance surface or object, for example in this paper it was a tree branch. The properties and shape of this object can change over the fault duration leading to changing fault characteristics as the fault progresses.

As shown in Fig. 8, in our experiments we have observed that there are four stages of a tree-branch HIF. From 0 to 10 s is the no-fault period, where the system is in steady-state. After $10 \mathrm{~s}$, the fault was initiated via the relay.

In the first stage after the fault was initiated (at 10s), the magnetic field in the $x$ and $z$ direction along with the phase $B$ voltage and current appear unchanged in the raw signals because the level of fault current is much lower than the load current. The voltage and current continue to show a linear relationship at this stage. No visual or audible phenomena were observed in the fault medium during this stage. The raw waveshapes of the early stage are therefore inseparable from the no fault stage.

In the second stage (from around 17.5s), fumes and an audible humming noise with sparks start to appear on the fault medium. The spark is a non-arcing discharge and the humming noise sounds like electrical hissing or sizzle. Therefore, the second stage as the sizzling stage. Some distortion of the Phase $B$ current signal is observable, whereas the voltage waveform remains unchanged. The V-I curve has therefore changed from linear to a slightly nonlinear shape. There is no readily visible change in the raw magnetic field measurements.

In the third stage, more significant variation in measured voltage, current and magnetic fields occur, where negative cycle arcing is present (from 18.95s). The Phase $B$ sinusoidal signals are distorted at peak positions due to the excess fault current. An intermittent transient arc feature appears on the negative half cycle of the current signal. During the arc, a significant drop in voltage distorts the sinusoidal voltage signal. Therefore, significant non-linearity between the voltage and current is visible on the negative half cycle in this third stage. In the magnetic field measurements, the $x$-axis sensor measurement is similar to the current sensor measurement, whereas the $z$-axis sensor shows less of the effect. Both visible and audible arc discharge happen in the fault box during this stage.

In the fourth and final stage (from 22.06s), a Stable Arc with half-cycle asymmetry appears in all current and magnetic field signals, and the sinusoidal voltage shape distorts on both the positive and negative cycle. Nonlinear voltage and current which is typical in others' studies of HIF at higher voltage levels [35], [36] is observable in the stable arc stage on both the positive and negative half cycle. Visible and audible arc discharge continues during this fourth stage and this ignites a fire.

As described above, arcing does not occur immediately following initiation of the fault. In fact it takes approximately 10 s after the fault was first initiated before some arcing was observed. In the first $8 \mathrm{~s}$ of the HIF, the presence of HIF is not readily observable in the raw data. Therefore, to explore the HIF characteristics further we have analysed the data using FFT and HT. 

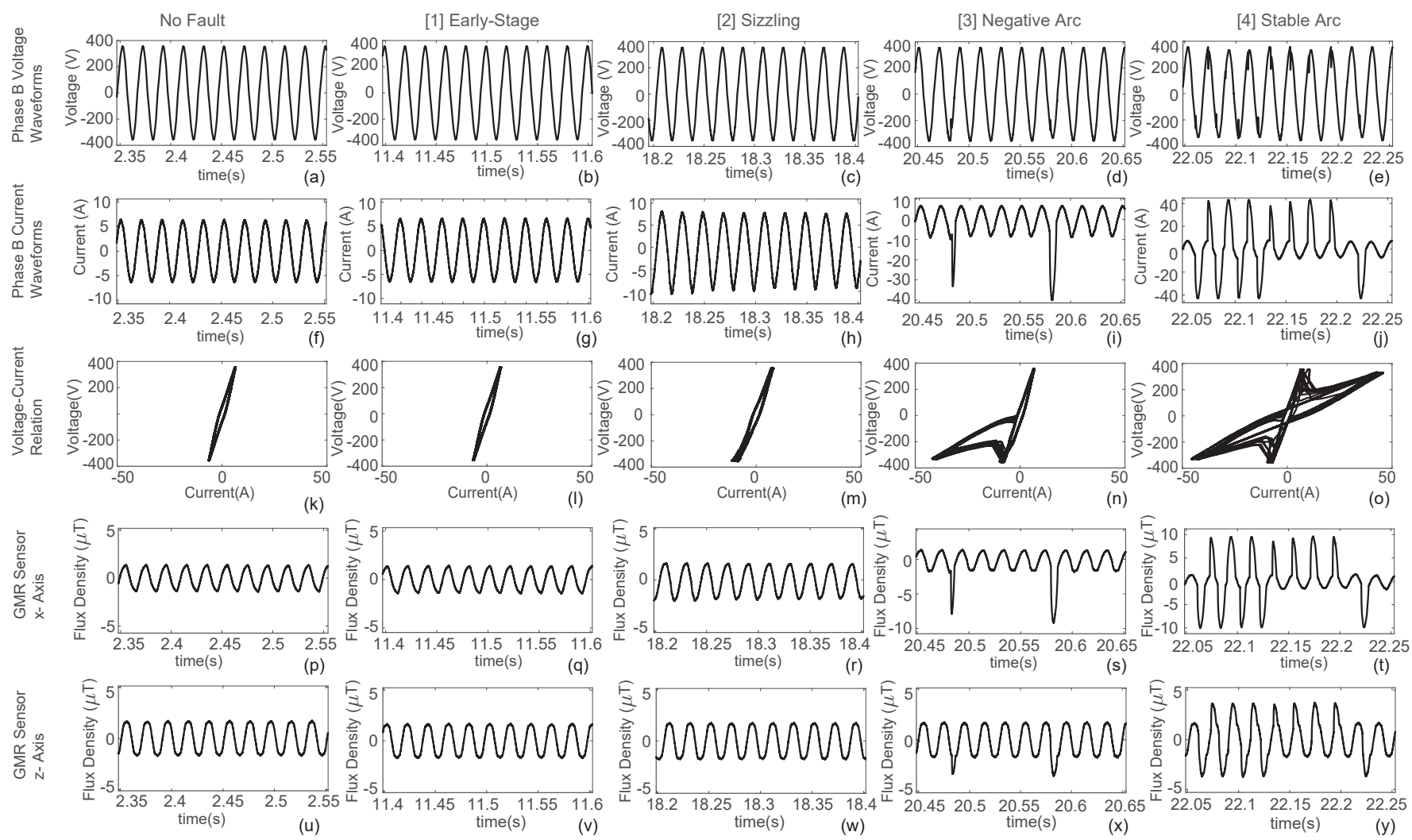

(n)
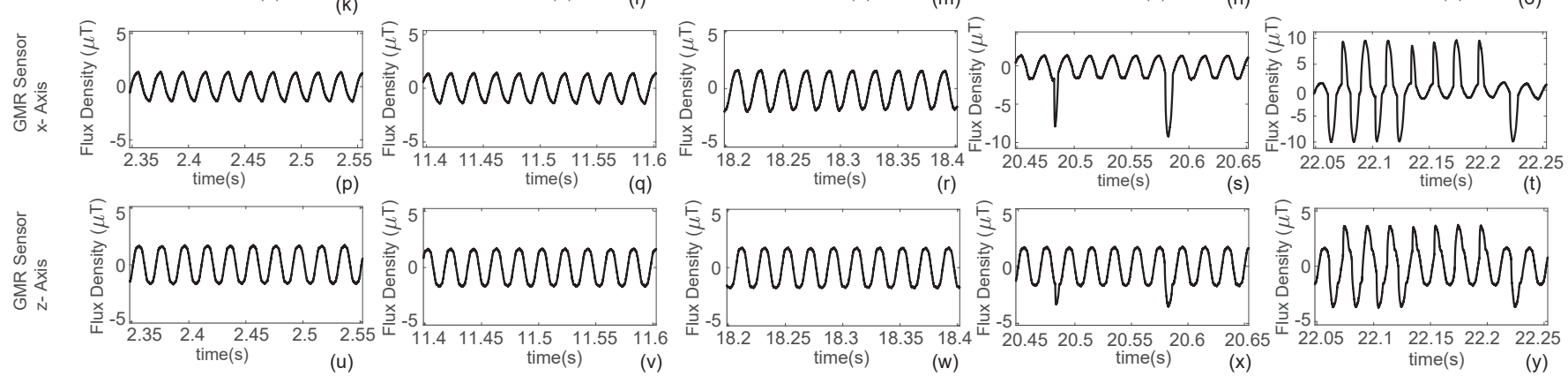

Fig. 8. Stages of HIF observed: in Voltage (Row 1), Current (Row 2), Current-Voltage characteristic (Row 3) and Magnetic field (Row 4, 5); Magnetic field signals are de-trended to eliminate the DC offset due to bias magnet.

\section{A. Characterization of Phase Current and Magnetic Signals in Frequency Domain}

Before applying the FFT, the dominant load frequency of $50 \mathrm{~Hz}$ was filtered from the Phase $B$ current and the magnetic sensor waveforms to focus on the high frequency content produced by the fault. A minimum order, Infinite Impulse Response (IIR) highpass $(90 \mathrm{~Hz}$ ) filter was applied to curtail the low-frequency components of the signals.

From the FFT data, we can observe in the normal nofault condition that both the current and magnetic sensors contain inherent electrical odd-harmonic noise, but of different magnitudes (Fig. 9 (a,f,k)). During the early-stage of the HIF (Fig. 9 (b,g,l)), there is a small change in the magnitude of these odd-harmonics, for both types of sensors. However, during the sizzling stage, new frequency components appear in the current sensor data, which are even harmonics. In the $x$-axis magnetic field measurements (Fig. 9 (h)), a significant increase in the odd harmonic frequencies occurs and the frequency content more closely resembles the current signals. During the later arcing stages (Fig. 9 (d,i,n,e,j,o)), a significant increase in magnitudes occur and new frequency components appear in both the current and magnetic field measurements. These are due to the intermittent and asymmetric nature of both the negative-only and stable arcing.

Overall, the magnitude of the new frequency components that appear during the latter fault stages are higher in the $x$ axis than the $z$-axis. The alternating magnetic fields are parallel to the $x$-axis and perpendicular to the $z$-axis direction of the sensor. Thus, the $x$-axis sensor can be considered as being more sensitive to magnetic field changes than the $z$-axis.

\section{B. Characterization of Phase Current and Magnetic Signals by Hilbert Transform}

Each row of Fig. 10 shows the breakdown of the IIR highpass $(90 \mathrm{~Hz})$ filtered signals into instantaneous amplitude and phase angle in the time domain, for each of the HIF stages.

The no-fault and early stages of the HIF do not show any significant $>90 \mathrm{~Hz}$ periodicity in either the current or magnetic field measurements, as the HT did not produce any visible magnitude envelope. However, the sizzling stage contains new periodicity, which generates a distinguishable magnitude envelope in the HT of the current and $x$-axis magnetic field signal. The arcing stage HT envelopes are visible in the current and both magnetic field measurements.

Similarly, the instantaneous phase angle highlights the frequency changes between the different stages. As the early stage does not contain much new frequency content, the filtered signals are essentially high frequency electrical noise. We therefore see no linear fluctuations in phase angle. In the sizzling stage, when new frequency content emerges $(>100 \mathrm{~Hz})$ the instantaneous phase angle starts to exhibit a more linear saw-tooth shape (although noisy). During the arcing stage, the signal contains high-magnitude low-frequency arc impulses disrupting the linear saw-tooth shape of the phase angle signal.

Regarding the GMR sensor measurements vs the current measurements, the HT magnitude envelopes during the sizzling stage are clear in the $x$-axis but less so than the current measurements. However, the $z$-axis magnetic field measurements are not as clear (Fig. 10 (m)). During the arcing stages of the HIF, the HT of the current and $x$-axis magnetic sensor measurements are very similar and the HT 

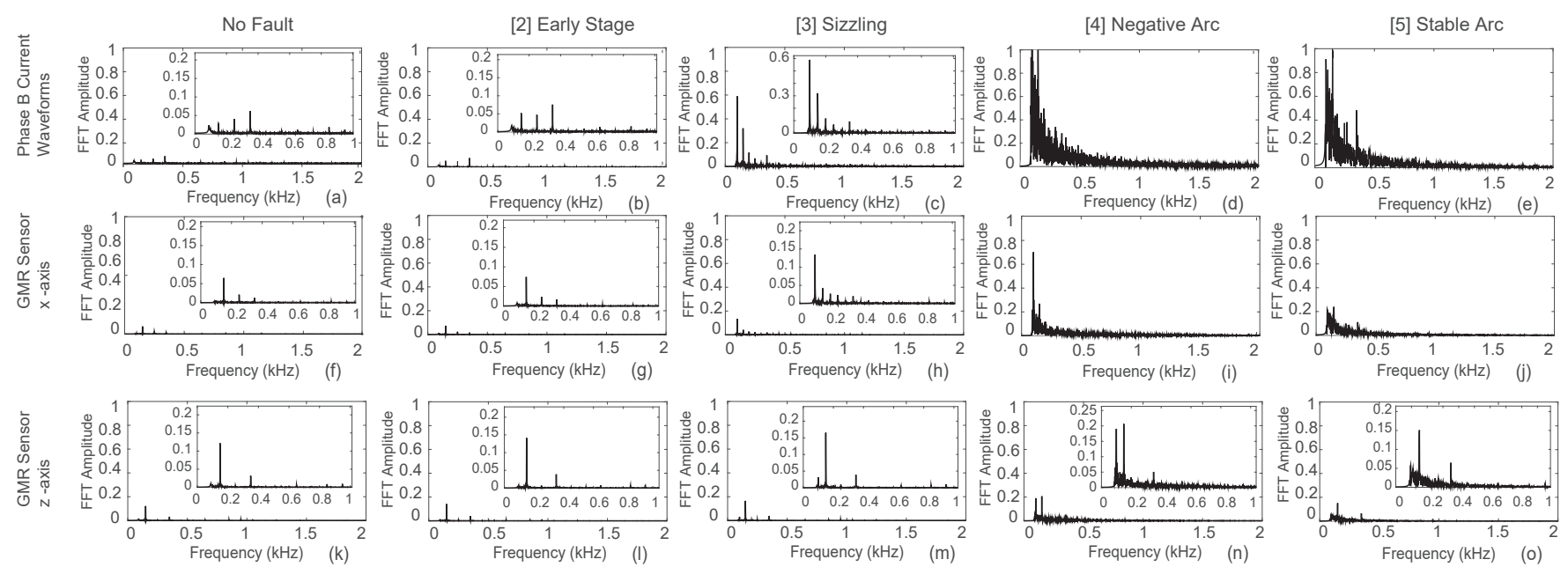

Fig. 9. Frequency Spectrum of different stages Current (Row 1) and Magnetic signals (Row 2 and 3) during the HIF stages: Frequency range (100Hz - 1kHz) insets.

algorithm has accurately localized the ignition and quenching moments during the negative arcing stage, for example as shown between 20.45 and 20.65s (Fig. 10 (d, i, n)).

\section{Fault Path Surface Phenomena}

As shown in preceding sections, some interesting new stages in the progression of an HIF fault were observed in these experiments, however the early stages of the HIF were not readily observable in the raw or transformed measurements. The $50 \mathrm{~Hz}$ load current present on the individual phase (Fig. 8) masks the effect of the low fault current during the HIF, for both the Hall-effect current sensor measurements and the GMR magnetic field measurements. Therefore, the early-stage, and sizzling stages of the HIF generate almost identical phase $B$ current and magnetic field (Fig. 8) waveform shapes to the no-fault stage.

We therefore investigated in more detail the phenomena occurring in fault path itself during the different stages of the HIF. This was using several additional sensors placed downstream of the isolation transformer. As seen in Fig. 11, the distinct characteristics of each of the stages during the HIF fault are more visually distinguishable in the fault path current waveform.

As the name HIF implies, the high-impedance of the fault medium only allows a small amount of current to flow. The average resistance of the tree branch before the fault is initiated was calculated (from voltage and current measurements) to be between $14 \mathrm{k} \Omega$ to $12 \mathrm{k} \Omega$. During the early stage of the HIF this calculated resistance drops to between $6.5 \mathrm{k} \Omega$ and $2.3 \mathrm{k} \Omega$. We see only 0.35 A (RMS) fault current (Fig. 11 (b)) and the wave shape resembles the saw-tooth waveform. This signal during the early stage contains significant third harmonic components compared to even harmonics (100 and $200 \mathrm{~Hz}$ ). A periodic shape gradually builds up later in the early-stage, but is limited to within 0.707 A (RMS).

The sizzling stage physically generates small sparks with an audible humming noise. The fault current waveforms during this time are found to be oscillatory and almost resemble the fullwave rectified waveform (Fig. 11 (c)). There are several valley points in this sizzling stage waveform. In the sizzling period, the level of peak current in the negative half-cycle is greater than its positive counterpart, $3 \mathrm{~A}$ versus $2 \mathrm{~A}$. The calculated tree branch resistance fluctuates between $0.4 \mathrm{k} \Omega$ and $0.2 \mathrm{k} \Omega$, which is significantly lower resistance than in the early stage. This waveshape in the sizzling stage is only observable in the Phase $B$ current and magnetic field measurements via HT and FFT analysis, where in the latter it produces the new and significant even harmonics.

The sizzling stage is an early indication of imminent arc discharge. During the sizzling stage, the tree branch resistance gradually decreases. The current flowing through the tree branch alters the chemical properties and produces a carbon track [37]. The carbon track influences the breakdown and arc discharge.

The negative arc stage is a breakdown state that developed during the sizzling period. As observable in the snapshot in figure 11 (d), the negative arc impulses are interspersed with sizzling. The presence of the two phenomena (sizzling and negative arcing) significantly shifts the level of odd and even harmonic frequency components in the FFT analysis of the phase $B$ current and GMR magnetic field measurements.

In the final steady or stable arc stage (Fig. 11 (e)), the arc waveform contains a half-cycle spark gap, and arc re-strike and extinction events can readily be observed in the data. Unlike the negative arc stage, in the steady arc stage the arc was observed to restrike for several successive cycles. It should be noted that in this study we have conducted HIF experiments in a controlled environment, where the bare conductor is continuously in contact with the tree branch (Fig. 5 (b)). In real-world scenarios, more occasional contact of a conductor with a tree branch may occur which would likely produce a more intermittent arc [38].

\section{Discussion}

In this study, we have experimentally analysed the behaviour of a tree-branch HIF in a 400 volt network physical test facility. The HIF events have unique current and magnetic field signatures, which will allow the presence of a fault to be differentiated from normal behaviour of a network. 

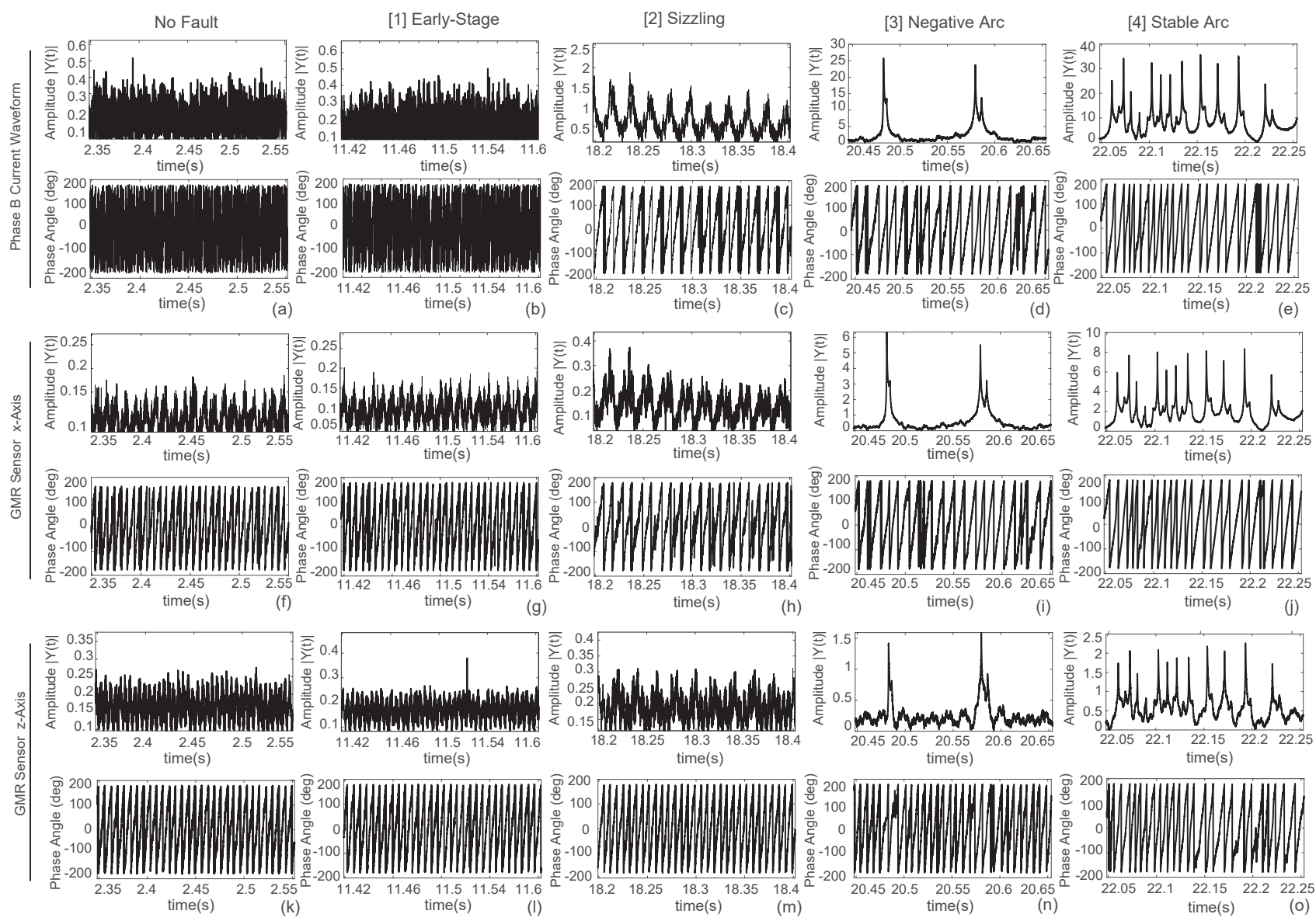

Fig. 10. Hilbert Transformation of phase current and magnetic field measurements during the HIF stages. The periodic magnitude envelope in the sizzling stage reveals the presence of weak non-linear and non-stationary behaviour in the phase current and magnetic sensor data.
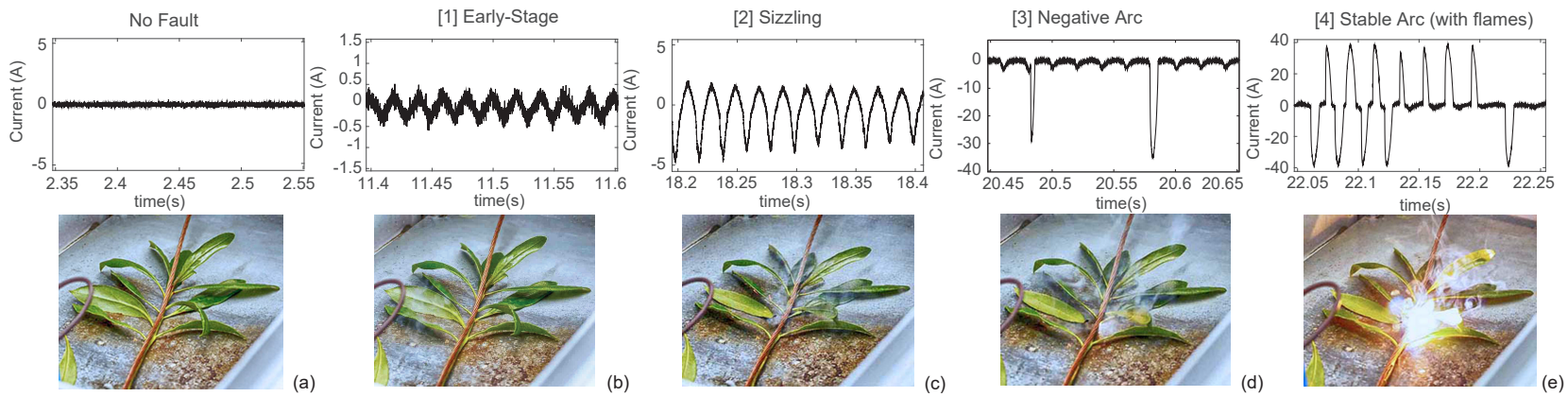

Fig. 11. Fault path current during HIF (a) No fault period; (b) Early-stage; (c) Sizzling stage; (d) Negative Arc stage; (e) Stable Arc stage. A slight swell is observed in the current waveform (d). The swell is due to the capacitance development between tree branch and metal plate. After initializing the HIF test, the current tries to pass through the tree branch to the metal surface. The nonlinear resistance of the tree branch impedes the current flow and as shown in the results in the later stages, produces an arc discharge. Snapshots during the experiment shown on the 2nd Row highlight that in the: Early-Stage, current starts to flow through test object; Sizzling stage fumes and gradual sparks are visible; Arc stage: audible and visible arc discharge that sets fire to the branch resulting in flames.

For a tree branch HIF, our experiment in a $400 \mathrm{~V}$ network simulator has highlighted two stages (early-stage followed by a periodic sizzling stage) preceding the expected known characteristic HIF behaviour of arcing. Using a current sensor connected to the fault path itself, we have observed the dynamic progression of HIF in detail. Previously in [39], $\mathrm{HIF}$ experiments at $11 \mathrm{kV}$ or higher have only identified the asymmetric, non-linearity between voltage-current and the intermittent nature of the HIF, which we also observe in the later stages. However, the $400 \mathrm{~V}$ HIF performed with a green tree branch as the surface material exhibited several build-up stages before going to steady-state arcing.

Unlike in the final arcing stages of the HIF, which are readily observable across all the raw measurements (voltage, current and magnetic field), in the initial stages of the HIF, the $50 \mathrm{~Hz}$ load current obscures the fault in the Phase current and magnetic field measurements. However, a low rank structure or intra-wave frequency modulation [40] is present in the fault path current (Fig. $11(\mathrm{~b}, \mathrm{c})$ ) in these initial early-stage and sizzling stages. These fault current features present in the early stages will therefore need to be extracted from Phase currents or magnetic field measurements via suitable signal processing 
algorithms in order for HIF faults to be detected during these early stages.

From the FFT analysis (Fig. 9), it is apparent that a treebranch based HIF contain increased frequency content within the frequency range $100 \mathrm{~Hz}$ to $1 \mathrm{kHz}$, with the $\mathrm{HIF}$ arcing stages, producing a significant energy burst.

The application of the HT to the raw measurements allowed a quantitative evaluation of the different stages of HIF faults from the current and magnetic measurements. The instantaneous frequency amplitude output of an HT shows the variation of inter-wave frequency modulation over time. During the sizzling stage of the HIF, the weak non-linear nonstationary fault current signal, which could not be seen clearly in the raw phase current or magnetic field signals is revealed by the HT. The HT also shows that the sizzling fault current signal is locally in-homogeneous [41], which means that the FFT analysis is less suitable as a basis for fault detection. During the negative arcing stage, the frequency doubling is distinguishable with the arc ignition and quenching moments localized in time by the HT. The HT phase-amplitude diagram or unwrapped phase function shows the presence of transients as it becomes continuous and almost smooth. The HT gives a good physical interpretation of HIF sizzling and arcing state in the time domain. However, further studies are needed to evaluate the effectiveness of Hilbert Transform as the basis for reliable detection and classification of HIF.

It would be interesting to model the fault and network event features extracted in the time-frequency domain via machine learning algorithms. The features we have observed exhibit different patterns [42], [43], which might be automatically recognized using Machine Learning algorithms. The classification of faults and typical network events, particularly during the challenging early-stage would require a real-world database. The experimental methodology used to conduct fault and network event experiments presented in this paper, has the potential to generate such a real-world experimental database.

Our study has allowed us to compare the fault characteristics observable with non-contact GMR sensors versus a Hall effect current sensor integrated with the phase conductors. The behaviour was able to be observed with both. The behaviour of HIF faults (from sizzling stage onwards) are all still identifiable in the non- contact GMR sensor measurements with considerable detail. In the magnetic field measurements, the $x$-axis of the GMR sensor were however found to be more sensitive to the transition of faults compared to $z$-axis, because the magnetic field relating to the fault is more predominantly parallel to the $x$-direction.

\section{A. Advantages of GMR sensors for industry}

Traditionally, voltage and current measurements in unidirectional low voltage $(400 \mathrm{~V})$ networks are rare. They are more common, in medium voltage networks, where the financial value of the network equipment being monitored is higher, giving a more acceptable business case for their use. However, due to the future bi-directional power supply scenario [44], there is an increasing need to monitor $400 \mathrm{~V}$ network as low or high impedance faults under this scenario will incur a cost to both network operators and consumers. Furthermore, installation of a widespread monitoring solution would enable more effective fault detection.

Most of the traditional monitoring sensors (e.g., CTs and PTs) in NZ are in either substations or switching stations, and for distribution networks more widespread deployment of integrated current sensors are cost-prohibitive. In contrast, GMR sensors are an attractive proposition for more widespread monitoring because of their broad bandwidth, low power consumption, miniature size, ability to digitally interface with intelligent systems and low cost. Hall effect sensors have traditionally been used for magnetic field measurement, however, they are susceptible to saturation due to the high permeability of their core [16]. Therefore, we are developing a widespread monitoring solution for distribution network using a non-contact GMR sensors. For practical evaluation, we are currently in contact with our local distribution companies (Wellington Electricity, North Power) and will install a prototype of GMR-based monitoring solution in a $400 \mathrm{~V}$ distribution network.

\section{CONCLUSION}

This work has revealed the differentiating characteristics of HIF in $400 \mathrm{~V}$ distribution networks. Real-world data was collected through a purpose-built $400 \mathrm{~V}$ overhead lines test facility. Conventional Hall-effect current and state-of-the-art GMR magnetic sensors captured the current and magnetic behaviour in the test facility. Distinct signatures of faults that will allow differentiation of them from normal network events were observed using both sensors.

Two new stages of HIF faults, i.e. an early followed by sizzling state, have been identified via current and magnetic field measurements. These states persisted for a reasonable period of time before arcing occurred. It would be desirable to detect HIF faults in real networks during these early stages, but they cannot be readily observed in raw current or magnetic field measurements. However, suitable signal processing techniques can reveal the characteristic features of these pre-arcing states.

As an alternative to current sensors integrated with the phase conductors, the properties of the GMR sensor make it well suited for widespread and non-contact monitoring of distribution overhead lines. However, there is a lack of practical data to evaluate the response of GMR sensor in the overhead line monitoring application. Our work therefore has advanced the knowledge regarding the application of GMR sensors to overhead lines monitoring. The magnetic field signals captured were found to be responsive to the dynamic behaviour of faults and load changes.

In the future, we will model the extracted characteristics of faults and normal system events from the GMR sensor data via Deep Learning (DL) algorithms towards classification of faults.

\section{APPENDIX \\ Negative ArC Phenomenon}

The negative half-cycle only arcing stage has not been observed by other researchers into HIF, so it deserves an explanation. Details are available in [45]. 


\section{REFERENCES}

[1] E. Vaahedi, Practical Power System Operation. John Wiley \& Sons, 2014, vol. 9781118394.

[2] J. Tengdin, R. Westfall, and K. Stephan, "High Impedance Fault Detection Technology Report of PSRC Working Group D15 March,” Tech. Rep., 1996.

[3] Maurice Blackburn Lawyers, "Last Black Saturday bushfire class action settles on steps of Court," Feb 2015. [Online]. Available: https://www.mauriceblackburn.com.au/about/media-centre/mediastatements/2015/last-black-saturday-bushfire-class-action-settles-onsteps-of-court/

[4] Abradee-Brazilian Association of Electricity Utilities, "9th National Week of Population Safety with Electricity," Tech. Rep. Rep no.11082014, 2015.

[5] N. Kularatna, A. S. Ross, J. Fernando, and S. James, "Design of Surge Protectors With a Practical Approach," in Design of Transient Protection Systems. Elsevier, Jan 2018, pp. 129-151.

[6] S. KhundmirT, "Introduction to Power Quality Monitoring," Tech. Rep., Jan 2015 .

[7] R. D. Christie, H. Zadehgol, and M. M. Habib, "High Impedance Fault Detection in Low Voltage Networks," IEEE Transactions on Power Delivery, vol. 8, no. 4, pp. 1829-1836, 1993.

[8] T. A. Kawady, "Close accord on characterizing and detecting arcing faults in LV distribution networks," in IEEE PES General Meeting, PES 2010, 2010, pp. 1-8.

[9] C. R. Ozansoy and D. P. S. Gomes, "Volatility Diagnosis in Phase-toPhase Fault Detection for Branch across Wire Faults," IEEE Transactions on Power Delivery, pp. 1-1, 2020.

[10] S. Kandanaarachchi, N. Anantharama, and M. A. Munoz, "Early detection of vegetation ignition due to powerline faults," IEEE Transactions on Power Delivery, p. 1, 2020.

[11] M. Wei, W. Liu, F. Shi, Z. Jin, H. Zhang, and W. Chen, "DistortionControllable Arc Modelling for High Impedance Arc Fault at the Distribution Network," IEEE Transactions on Power Delivery, p. 1, 2020.

[12] A. Cataliotti, D. Di Cara, A. E. Emanuel, and S. Nuccio, "Characterization of current transformers in the presence of harmonic distortion," in Conference Record - IEEE Instrumentation and Measurement Technology Conference. IEEE, 2008, pp. 2074-2078.

[13] K. J. Ferreira and A. E. Emanuel, "A noninvasive technique for fault detection and location," IEEE Transactions on Power Delivery, vol. 25, no. 4, pp. 3024-3034, 2010.

[14] N. Locatelli and V. Cros, "Basic Spintronic Transport Phenomena," Introduction to Magnetic Random-Access Memory, pp. 1-28, 2016.

[15] M. N. Baibich, J. M. Broto, A. Fert, F. N. Van Dau, F. Petroff, P. Eitenne, G. Creuzet, A. Friederich, and J. Chazelas, "Giant magnetoresistance of $(001) \mathrm{Fe} /(001) \mathrm{Cr}$ magnetic superlattices," Physical Review Letters, vol. 61, no. 21, pp. 2472-2475, 1988.

[16] P. N. Ayambire, Q. Huang, D. Cai, O. Bamisile, and P. O. Kwasi Anane, "Real-time and contactless initial current traveling wave measurement for overhead transmission line fault detection based on tunnel magnetoresistive sensors," Electric Power Systems Research, vol. 187, p. 106508, 2020.

[17] M. Sarlak and S. M. Shahrtash, "High-impedance faulted branch identification using magnetic-field signature analysis," IEEE Transactions on Power Delivery, vol. 28, no. 1, pp. 67-74, 2013.

[18] A. F. Sultan, G. W. Swift, and D. J. Fedirchuk, "Detecting arcing downed-wires using fault current flicker and half-cycle asymmetry," IEEE Transactions on Power Delivery, vol. 9, no. 1, pp. 461-470, 1994.

[19] A. I. Sifat, J. Bailey, K. Hamilton, F. J. McFadden, R. Rayudu, and A. Hunze, "A Facility for Physical Simulation of High Impedance Faults in Low Voltage Networks," in IEEE Power and Energy Society General Meeting, vol. 2019-Aug, 2019, pp. 1-5.

[20] John J. Grainger, Power System Analysis. McGraw-Hill, 2003.

[21] Horizon-Networks, "Aseet Management Plan 2019-26," Tech. Rep., 2020.

[22] LEM, "Current Transducer HO-NP Series," LEM, Tech. Rep., 2018. [Online]. Available: https://www.lem.com/sites/default/files/products_datasheets/ho-np0000_series.pdf

[23] National Instruments, "NI Multifunction I/O Device," 2019. [Online]. Available: http://www.ni.com/en-nz/shop/select/multifunction-io-device

[24] — "DAQ Synchronisation." [Online]. Available: http://www.ni.com/product-documentation/54461/en/
[25] NVE Corporation, "GMR - AA and AB-Series Ana$\log$ Sensors," p. 16, 2015. [Online]. Available: https://www.nve.com/Downloads/analog_catalog.pdf

[26] A. Mamishev, R. Nevels, and B. Russell, "Effects of conductor sag on spatial distribution of power line magnetic field," IEEE Transactions on Power Delivery, vol. 11, no. 3, pp. 1571-1576, 1996.

[27] Q. Huang, S. Jing, J. Yi, and W. Zhen, "New types of sensors for smart grid," Innovative Testing and Measurement Solutions for Smart Grid, pp. 11-80.

[28] A. H. Detti, S. Jemei, S. Morando, and N. Y. Steiner, "Classification Based Method Using Fast Fourier Transform (FFT) and Total Harmonic Distortion (THD) Dedicated to Proton Exchange Membrane Fuel Cell (PEMFC) Diagnosis," in 2017 IEEE Vehicle Power and Propulsion Conference (VPPC), 2017, pp. 1-6.

[29] J. O. S. Iii, "Mathematics of the Discrete Fourier Transform ( DFT )," Identity, 2002

[30] R. Banerjee, Prasanta Kumar and Butterfield, "Boundary element methods in engineering course," Engineering Analysis, vol. 1, no. 1, pp. 61-62, 2003.

[31] T. Cui, X. Dong, Z. Bo, and A. Juszczyk, "Hilbert-transform-based transient/intermittent earth fault detection in noneffectively grounded distribution systems," IEEE Transactions on Power Delivery, vol. 26, no. 1, pp. 143-151, 2011.

[32] Digital Signal Processing Committee, Programs for Digital Signal Processing. IEEE Press, 1979.

[33] J. F. Claerbout, Fundamentals of geophysical data processing. Citeseer, 1985.

[34] T. E. Conturo and G. D. Smith, "Signal-to-noise in phase angle reconstruction: Dynamic range extension using phase reference offsets," Magnetic Resonance in Medicine, vol. 15, no. 3, pp. 420-437, 1990.

[35] C. Gonzalez, J. Tant, J. G. Germain, T. De Rybel, and J. Driesen, "Directional, high-impedance fault detection in isolated neutral distribution grids," IEEE Transactions on Power Delivery, vol. 33, no. 5, pp. 24742483, 2018.

[36] S. Hamid Mortazavi, Z. Moravej, and S. Mohammad Shahrtash, "A Searching Based Method for Locating High Impedance Arcing Fault in Distribution Networks," IEEE Transactions on Power Delivery, vol. 34, no. 2, pp. 438-447, 2019.

[37] T. A. Short, Electric Power Distribution Handbook. CRC Press, 2018.

[38] B. M. Aucoin and B. D. Russell, "Distribution High Impedance Fault Detection Utilizing High Frequency Current Components," IEEE Transactions on Power Apparatus and Systems, vol. PAS-101, no. 6, pp. 15961606, 1982.

[39] A. Ghaderi, H. L. Ginn, and H. A. Mohammadpour, "High impedance fault detection: A review," Electric Power Systems Research, vol. 143, pp. 376-388, Feb 2017.

[40] P. Zhou, M. Du, S. Chen, Q. He, Z. Peng, and W. Zhang, "Study on intra-wave frequency modulation phenomenon in detection of rubimpact fault," Mechanical Systems and Signal Processing, vol. 122, pp. 342-363, May 2019.

[41] N. E. Huang, Z. Shen, S. R. Long, M. C. Wu, H. H. Shih, Q. Zheng, N.C. Yen, C. C. Tung, and H. H. Liu, "The empirical mode decomposition and the Hilbert spectrum for nonlinear and non-stationary time series analysis," Proceedings of the Royal Society of London. Series A: Mathematical, Physical and Engineering Sciences, vol. 454, no. 1971, pp. 903-995, 1998.

[42] T. M. Lai, L. A. Snider, E. Lo, and D. Sutanto, "High-impedance fault detection using discrete wavelet transform and frequency range and RMS conversion," IEEE Transactions on Power Delivery, vol. 20, no. 1, pp. 397-407, 2005

[43] D. P. Gomes, C. Ozansoy, and A. Ulhaq, "High-Sensitivity Vegetation High-Impedance Fault Detection Based on Signal's High-Frequency Contents," IEEE Transactions on Power Delivery, vol. 33, no. 3, pp. 1398-1407, 2018.

[44] N. Patel, D. Porwal, A. K. Bhoi, D. P. Kothari, and A. Kalam, "An Overview on Structural Advancements in Conventional Power System with Renewable Energy Integration and Role of Smart Grids in Future Power Corridors," in Green Energy and Technology, A. K. Bhoi, K. S. Sherpa, A. Kalam, and G.-S. Chae, Eds. Singapore: Springer Singapore, 2020, pp. 1-15.

[45] A. I. Sifat, J. Bailey, F. J. S. McFadden, R. Rayudu, and A. Hunze, "Negative Arc phenomenon." [Online]. Available: https://bit.ly/2zyqlk7 


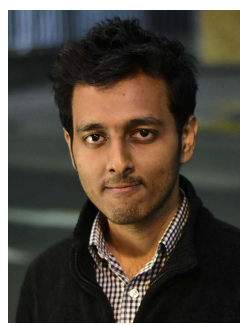

Anwarul Islam Sifat received B.Sc. in Electriable Energy Technology from Stamford University Bangladesh (2013) and the University of Dhaka (2017), Bangladesh, respectively. He is currently pursuing a PhD degree at the School of Engineering and Computer Science (ECS), Victoria University of Wellington. His current research project is on low voltage distribution power sector faults identification, classification, and location using magnetoresistive sensors. He has research and work interests in Power system engineering, applied deep learning, and embedded systems. cal/Electronic Engineering and M.Sc. in Renew-

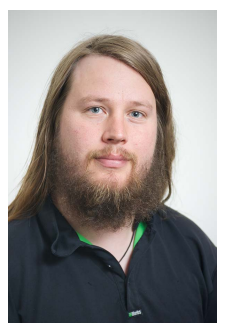

Joseph Bailey received a B.E (Hons) from Canterbury University in 2011 and an M.E from Victoria University of Wellington in 2015. He joined Industrial Research Limited as a junior engineer in 2011 and now works at Victoria University of Wellington as an Engineer. He is currently working on his $\mathrm{PhD}$ in Engineering at Victoria University of Wellington. His current research interests include magnetic sensing technology and non-destructive testing.

Arvid Hunze has 20 years' experience in applied the industrial, commercial, and academic sectors in both Australia and New Zealand. She is currently a Principal Engineer with the Robinson Research Institute, Victoria University of Wellington (VUW), Wellington, New Zealand, and is also with the Smart Power and Renewable Energy Systems Group, VUW. Her current research platform is in predictive data analytics and modelling for decision-making, control, and optimisation for a range of industry applications. One project area is sensor development and application of predictive modelling and data analytics to improve the operation of the electricity distribution network and extend the life of the distribution infrastructure. This includes detection of overhead line electrical faults, and prediction of transformer thermal behaviour and acceptable overload conditions. Another project area is sensing, perception and control approaches for safe behaviour of adaptive learning robots. Over her career she has received four Best Paper awards for her research and in 2013 one of her earliest research publications was republished in a compilation of papers recognized as the most important findings and insights reported over the past five decades in aluminium smelting.

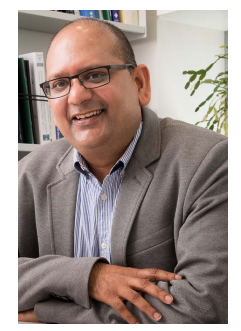

Ramesh Rayudu (S'94-SM'11) received the B.E. (First Class Distinction) degree from Osmania University, Hyderabad, India, in 1993, the M.E. degree from the University of Canterbury, Christchurch, New Zealand, 1993, and the Ph.D. degree in AI and power systems engineering from Lincoln University, Lincoln, New Zealand, in 2001. He has more than 15 years of industrial work experience both in India and New Zealand. He is also involved in consultation work for several international firms in New Zealand, Australia, Singapore, Malaysia, India, and the USA. He is currently an Associate Professor with the Victoria University of Wellington, Wellington, New Zealand. He has authored or co-authored publications for journals, invited articles, and papers for magazines and conferences and holds three patents. His current research interests include power systems engineering, renewable energy systems, artificial intelligence (AI) applications, health monitoring, and energy harvesting. He is the recipient of three Best Paper and four Best Presentation Awards. He is an Associate Editor of three international journals. He was co-awarded the prestigious IPENZ 1999 Fulton Downer Silver Medal for his work. He is active in IEEE activities and is currently the Chair of local PES Chapter. He was also the General Co-Chair for the 2017 IEEE PES Innovative Smart Grid Technologies Asia conference and 2020 Image and Vision Computing New Zealand (IVCNZ) conference, New Zealand. research and development at Siemens Cooperative Technology and Renewable Energy business unit, New Zealand Crown Research Institute IRL, and Victoria University of Wellington. His work includes materials and applications for organic electronics, organic light emitting diodes, sensors, nondestructive testing, electricity network asset management, technical project management and securing science funding from different sources. He published over 30 peer-reviewed journal articles and confer-

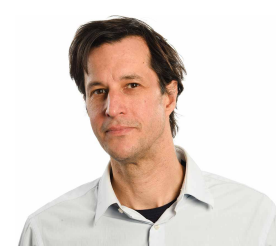

ence proceedings and more than 100 patents in 27 patent families in organic electronics, sensors and non-destructive testing. 